\title{
Lecciones aprendidas a partir de la experiencia de la aplicación del aprendizaje basado en proyectos en el Grado en Ingeniería Química de la UPV
}

\author{
García-Fayos B. ${ }^{\text {a }}$, Sancho M. ${ }^{\text {, }}$, Arnal J.M. ${ }^{\text {c }}$ \\ ${ }^{a}$ Universitat Politècnica de València (UPV). Chemical and Nuclear Engineering Department. Camino \\ de Vera s/n. Building 5L. 46022 Valencia (Spain). E-mail: beagarfa@iqn.upv.es,

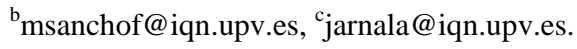

\begin{abstract}
Resumen
El aprendizaje basado en proyectos $(A B P)$ es una metodología docente consistente en proponer un proyecto con solución abierta que los alumnos deben resolver. Para ello, deben adquirir una mayor responsabilidad de su propio aprendizaje, reflexionando y adoptando decisiones en el grupo para resolver el proyecto. Con esta metodología se logra un aprendizaje integrador y el desarrollo de competencias tales como trabajo en grupo o gestión del tiempo, útiles en su profesión. Un grupo de profesores, llevamos desde el curso 2004-2005 aplicando esta metodología en asignaturas experimentales obligatorias de Ingeniería Química en la UPV a través del desarrollo de un proyecto de diseño. La implantación del EEES, supuso la adaptación del ABP para su desarrollo en la asignatura de Experimentación en Ingeniería Química III de Grado. Este trabajo relata el planteamiento para la implementación de esta metodología, así como las dificultades encontradas y las lecciones aprendidas, fruto de la experiencia adquirida en tres cursos académicos. Para ello, se analizarán los datos recogidos a través de encuestas anónimas realizadas a los alumnos, así como las conclusiones de las reuniones de "focus group" realizadas. Las conclusiones obtenidas servirán para mejorar la aplicación de esta metodología en los próximos cursos académicos y de guía para otras asignaturas de la titulación.
\end{abstract}

Palabras clave: ABP, ingeniería química, proyecto, experimentación, diseño 
Lecciones aprendidas a partir de la experiencia de la aplicación del aprendizaje basado en proyectos en el Grado en Ingeniería Química de la UPV

\section{Introducción}

El Ingeniero Químico se pretende que sea un profesional con amplios conocimientos en la rama de la Química y al mismo tiempo con capacidad para concebir, proyectar y controlar las industrias de proceso y todos aquellos elementos que las componen. De acuerdo con ello, su formación debe comprender tanto la adquisición de conocimientos como la puesta en práctica de los mismos. Asimismo, un ingeniero debe ser capaz de resolver problemas reales, lo que supone realizar un estudio cuantitativo lo más exacto posible de los parámetros implicados en el mismo, a lo que contribuye la experimentación. Por ello, tradicionalmente, los planes de estudio del ingeniero químico han incluido una parte importante de experiencia práctica en laboratorio, la cual es esencial para entender los fundamentos de la Ingeniería química (Peñas et al., 2006).

A raíz de la adaptación de las universidades españolas al Espacio Europeo de Educación Superior, las titulaciones de Ingeniería Química existentes se transformaron en las actuales titulaciones de Grado y Máster en Ingeniería Química, dando continuidad al planteamiento inicial establecido en relación a la experimentación, existiendo asignaturas específicas como materia obligatoria. El análisis de los planes de estudio actualmente vigentes de Grado muestran al menos dos asignaturas cuatrimestrales de 6 créditos ECTS que se imparten entre el segundo y cuarto curso de todos los planes de estudios. En el caso de la UPV, la titulación de Ingeniería Química anterior al Grado contenía cuatro asignaturas obligatorias de experimentación en las que se realizaban prácticas de laboratorio por grupos. En la última de esas asignaturas, a partir del curso 2004-2005, se llevó a cabo la implantación de la metodología del ABP, (Sancho et al., 2007; Sancho et al., 2009) lo que permitió relacionar las prácticas realizadas a través de un proyecto de diseño de una instalación industrial, con excelentes resultados (Arnal et al., 2012).

El ABP es una metodología de enseñanza-aprendizaje que se basa en plantear a los alumnos la realización de un proyecto, definido como un problema complejo de solución abierta, en el que es necesario la investigación, la reflexión y la toma de decisiones para su resolución (Blumenfled et al., 1991). El profesor actúa como guía en el proceso y no como principal fuente de información. Esta metodología proporciona a los alumnos la oportunidad de realizar un proyecto en un entorno de trabajo simulado (Kuntalp et al., 2010), lo que mejora su motivación al ver que el resultado final es un proyecto real. Además, facilita un aprendizaje autónomo e integrador, y el desarrollo de habilidades y destrezas tales como el trabajo en grupo, gestión de la información, pensamiento creativo o la toma de decisiones (De Miguel, 2005). 
Con la adaptación de la titulación al proceso de Bolonia, el número de asignaturas experimentales en el Grado se redujo a tres. A pesar de ello, se decidió trasladar el planteamiento de la metodología ABP a la última asignatura experimental de la titulación, "Experimentación en Ingeniería Química III" (Sancho et al., 2017). Este trabajo recoge el análisis de los resultados obtenidos tras la aplicación de la metodología en la asignatura a lo largo de tres cursos académicos y describe las estrategias adoptadas para continuar mejorando su implementación en los próximos cursos.

\section{Innovación metodológica}

Experimentación en Ingeniería Química III es una asignatura experimental de tercer curso cuatrimestre B de 4,5 créditos ECTS y es la última de las asignaturas experimentales del Grado en Ingeniería Química de la UPV.

La aplicación de la metodología del ABP se realiza en la Unidad Didáctica 1, que tiene por título "Diseño de un proceso industrial" y una duración de 10 semanas. En ella, los alumnos realizan prácticas de laboratorio que están relacionadas con las etapas del proceso productivo a diseñar, estudiando las variables que afectan a éste y recogiendo datos que se utilizarán posteriormente en el proyecto. En cada sesión, el alumno realiza un informe diario a modo de trabajo académico, pudiendo de esta forma dedicar el tiempo fuera del aula al desarrollo del proyecto. La elaboración del informe en el laboratorio es una metodología nueva para los alumnos, dado que en las asignaturas previas de experimentación, el informe se realiza mayoritariamente tras la finalización de la práctica. Para la resolución de las dudas del proyecto y obtención de feed-back sobre el desarrollo del mismo, los alumnos pueden consultar a los profesores concertando sesiones de tutorías. No obstante, en la planificación de la asignatura se incluye una sesión de tutorías grupal (semana 6) con el fin de tener al menos un punto de control de desarrollo del trabajo a lo largo del semestre. Finalmente, en la semana 10 se incluye una sesión para la defensa oral del proyecto. Las sesiones que incluye esta unidad y su relación con el proyecto se muestran en la Fig. 1.
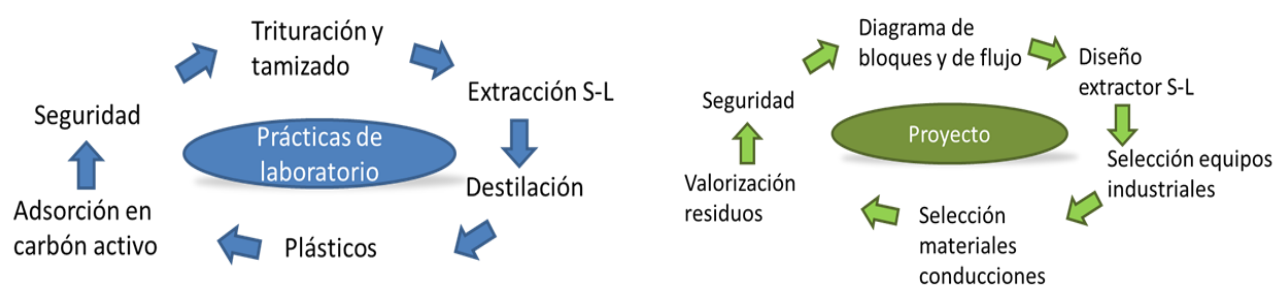

Fig. 1. Diagrama de relación de las practicas de laboratorio con el proyecto de diseño 
Lecciones aprendidas a partir de la experiencia de la aplicación del aprendizaje basado en proyectos en el Grado en Ingeniería Química de la UPV

La evaluación de la asignatura se realiza a través de los informes diarios de cada práctica realizados en el laboratorio (20\% de la nota final), el proyecto de diseño (30\%), el examen oral $(10 \%)$, que consiste en la presentación del proyecto, y el examen (10\%). El $30 \%$ restante corresponde a la unidad didáctica 2.

\section{Objetivo}

El objetivo de este trabajo es analizar la implementación del ABP en una asignatura experimental de GIQ y proporcionar recomendaciones para el éxito de su aplicación en el desarrollo de un proyecto de diseño.

\section{Metodología}

Para analizar la implementación del ABP en la asignatura en los tres últimos cursos (con un número de alumnos matriculados entre 64 y 81 ) se han utilizado distintas herramientas:

-Recopilación de la opinión del alumnado sobre la metodología implementada (contenidos, trabajo académico y tutorías): a través de una encuesta de opinión anónima de 7 preguntas (ver Tabla 1.1), con una escala de Likert de 4 niveles (A: Totalmente de acuerdo, B: Parcialmente de acuerdo, C: Parcialmente en desacuerdo, D: Totalmente en desacuerdo).

-Recopilación y procesado de las respuestas obtenidas en las reuniones de grupo. Cuando se han percibido desviaciones en el desarrollo de la asignatura, se han realizado reuniones de "focus group", entre los profesores y un representante de cada grupo de trabajo, para tratar diferentes aspectos sobre la asignatura.

Tabla 1.1. Preguntas de la encuesta de opinión sobre la metodología ABP implementada

\begin{tabular}{l}
\hline CONTENIDOS \\
1-Los contenidos tratados son de aplicación profesional \\
2-La asignatura permite relacionar y aplicar conocimientos de otras asignaturas \\
3-El número y tipo de prácticas de laboratorio han sido útiles para la realización del proyecto \\
\hline TRABAJO ACADÉMICO: INFORMES DE PRÁCTICAS \\
\hline 4-La realización de los informes durante la sesión de laboratorio mejora la calidad de los mismos \\
5-Realizar los informes en el laboratorio supone una descarga de trabajo significativa \\
\hline TUTORÍAS \\
6-Las tutorías han sido útiles para orientar la organización del proyecto y analizar la progresión a \\
lo largo del cuatrimestre \\
7-Considero que las sesiones de tutoría son indispensables para el desarrollo del proyecto con éxito \\
\hline
\end{tabular}




\section{Resultados y análisis}

\subsection{Análisis del nivel de satisfacción de los estudiantes}

Se analizará el nivel de satisfacción de los estudiantes con los contenidos impartidos, con la metodología de realización de informes en el laboratorio y con las tutorías.

\subsubsection{Contenidos}

En primer lugar se muestran los resultados sobre la opinión de los estudiantes al respecto de la selección de contenidos de la asignatura y su relación con el proyecto a desarrollar. En la Fig. 2 se muestran los resultados para cada curso académico (16-17, 17-18 y 18-19) y la pregunta que corresponde (preguntas 1, 2 y 3). Como se observa, en todos los cursos académicos la suma de las respuestas de total (A) y parcialmente de acuerdo (B) es superior al $90 \%$, en todas las preguntas planteadas. Por ello, se deduce que los alumnos están muy satisfechos con los contenidos de la asignatura porque: son de aplicación en su futuro profesional (pregunta 1); están relacionados con otras asignaturas de la titulación (pregunta 2); y, tal y como se desarrollan en las prácticas de laboratorio, ayudan al desarrollo del proyecto final (pregunta 3).

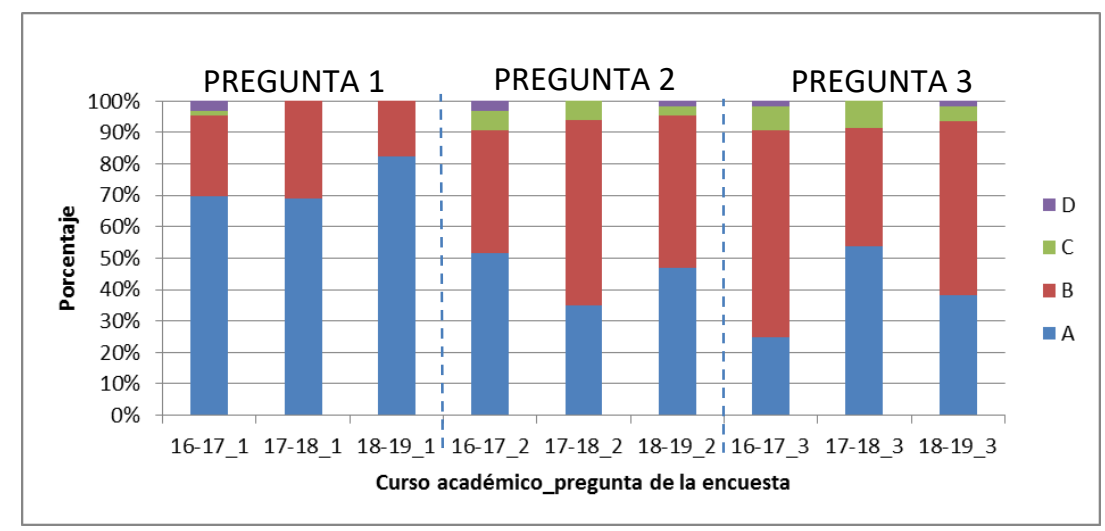

Fig. 2 Nivel de satisfacción con los contenidos de la asignatura

\subsubsection{Informes de laboratorio}

Respecto a los informes de laboratorio, los resultados se muestran en la Fig. 3. 


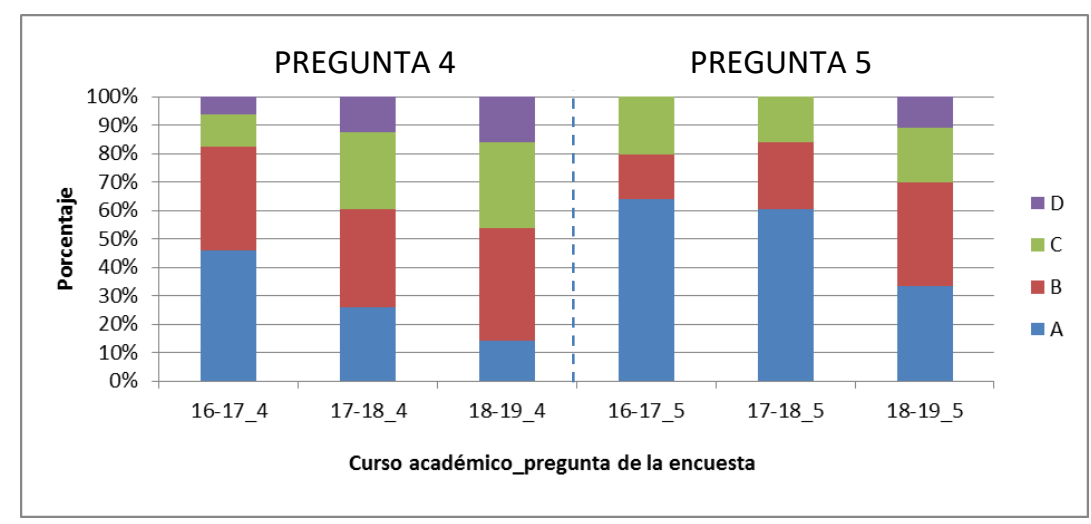

Fig. 3 Nivel de satisfacción con la metodología de realización de los informes de laboratorio

La opinión de los alumnos respecto del cambio de metodología que supone realizar los informes en el laboratorio muestra que el nivel de satisfacción es medio, ya que al menos el $50 \%$ de los alumnos están total o parcialmente de acuerdo en considerar que esta metodología ayuda a mejorar la calidad de los informes respecto a realizarlos fuera del laboratorio (pregunta 4).

Respecto a si realizar el informe en el laboratorio supone una descarga de trabajo fuera del aula (pregunta 5), la opinión de los alumnos es similar en los cursos 16-17 y 17-18 con, al menos, un $80 \%$ de los alumnos total y parcialmente de acuerdo, y un $70 \%$ en el curso 18 19. En este curso se observa que el porcentaje de alumnos totalmente de acuerdo y parcialmente de acuerdo disminuye un $10 \%$ y se incrementa en esa misma propoción en alumnos que están totalmente en desacuerdo.

Se debe tener en cuenta que los alumnos del curso 2016-2017 habían realizado informes en el laboratorio en la asignatura de experimenactión previa (en al menos dos prácicas), por lo que habían tenido la oportunidad de practicar este tipo de metodología, y por ello valoran positivamente la ventaja que supone. Los alumnos del curso 2017-2018 solo lo habían practicado en una sesión de laboratorio de la asignatura de experimentación previa y los alumnos del curso 2018-2019 es la primera vez que realizan los informes en el laboratorio, por lo que no están habituados a esta metodología. Por ello, esto puede suponerles más esfuerzo y lo valoran en consecuencia más negativamente. Este resultado provocó la reunión de "focus-group" con los alumnos del curso 2018-2019 con el fin de conocer con mayor detalle su opinión a este respecto.

\subsubsection{Tutorías}

Respecto a la utilidad de las tutorías para la organización del trabajo y el desarrollo del proyecto, los resultados de valoración se muestran en la Fig. 4. En ella se observa que hay una valoración positiva o muy positiva en el $90 \%$ de los casos. 
En concreto se observa que:

-La utilidad de las tutorías (pregunta 6) ha sido valorada muy positivamente por los alumnos. Al menos un $65 \%$ de los alumnos lo valoran muy positivamente (A), y entre positivo y muy positivamente (A y B) un $85 \%$ de los alumnos. No obstante, destaca el incremento del $10 \%$ de los alumnos que están parcialmente en desacuerdo con la utilidad de los tutorías en el curso académico 2018-2019.

-La necesidad de asistir a tutorías como herramienta indispensable para poder desarrollar el proyecto (pregunta 7) ha sido valorada en conjunto como muy necesaria (A) o necesaria (B) para el $95 \%$ de los alumnos, si bien es verdad que la opinión ha ido empeorando a medida que transcurren los cursos académicos, observándose un incremento del triple de alumnos que han pasado de muy satifechos a parcialmente satifechos del curso 2016-2017 al actual.

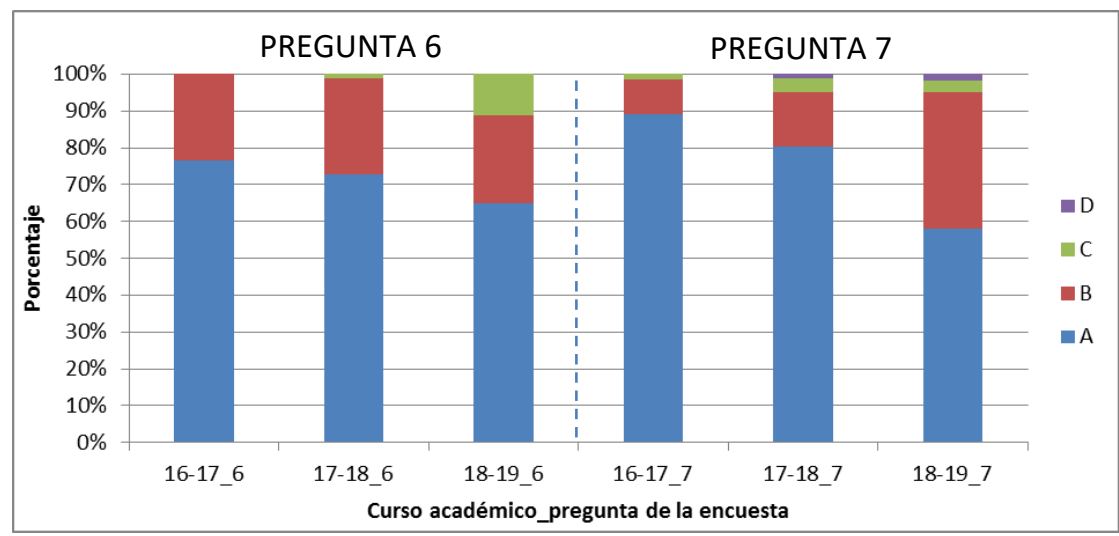

Fig. 4 Nivel de satisfacción con las tutorías

Por tanto, se observa una tendencia a la reducción de la satisfacción de las tutorías para la elaboración del proyecto en el último curso académico. Este emporamiento ha sido objeto de análisis en las reuniones de "focus-group", que han dado lugar a las conclusiones que se describen en el siguiente apartado y a analizar el uso de esta herramienta.

El gráfico de la Fig. 5 muestra la distribución de tutorías a lo largo del cuatrimestre y el número de tutorías por grupo en media. 
Lecciones aprendidas a partir de la experiencia de la aplicación del aprendizaje basado en proyectos en el Grado en Ingeniería Química de la UPV

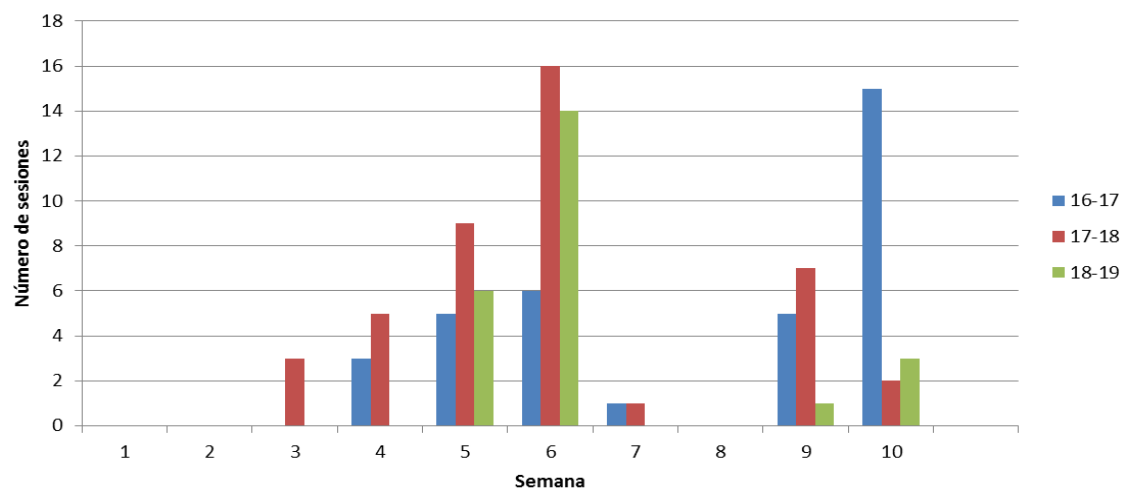

Fig. 5 Distribución de las tutorías a lo largo del cuatrimestre

En el registro de tutorías se observa que la asistencia a éstas comienza en la semana 3-4 tanto para el curso 16-17 como el 17-18, que coincide con la realización de la práctica que constituye el eje del diseño (Extracción S-L). Sin embargo, se aprecia un comienzo más tardío en el curso 18-19, situándose en la semana 5.

En el curso 16-17, se observó que aunque la asistencia a tutorías era progresiva, el máximo se concentraba en la semana 10 que era la de las tutorías grupales y la previa a la entrega del proyecto de diseño. Por ello, en los cursos siguientes se planificó la tutoría grupal a mitad del semestre para tener ese punto de control del trabajo con tiempo suficiente para seguir desarrollándolo a partir del feed-back proporcionado. Para los cursos siguientes se observa que el número de tutorías posee una distribución de campana de Gauss. Éste aumenta progresivamente hasta la semana 6 , que es donde se sitúa el máximo de la campana, coincidiendo con la tutoría grupal. En la semana 7-8 hay una disminución o ausencia total de asistencia a tutorías coincidiendo con una semana de exámenes parciales y un periodo de vacaciones, y se reanudan aunque con una menor intensidad hasta la entrega del trabajo en la semana 10.

Respecto a la media de sesiones por grupo, resaltar que, en media, los cursos 16-17 y 17-18 reflejan una asistencia de, al menos, 1.3 sesiones de tutoría por grupo para la resolución del diseño y en el curso 18-19, de 0.7 sesiones de tutoría por grupo. Esto significa que algunos grupos (en concreto un $50 \%$ de ellos) resolvieron el proyecto de forma autónoma sin la guía y la supervisión del profesor-tutor. Por tanto, este escaso uso de las tutorías y un comienzo más tarde de su uso para la orientación en el proyecto explicarían su peor valoración en el curso 2018-2019. 


\subsection{Dificultades detectadas y decisiones de mejora adoptadas}

La reunión de "focus-group" fue necesaria para el curso 2018-2019 debido a una serie de anomalías detectadas en el desarrollo del semestre y de los trabajos, en comparación con los cursos anteriores. Las cuestiones planteadas a los alumnos, y sus respuestas han sido recogidas en la Tabla 1.2, estructuradas por tema, aspectos positivos y aspectos de mejora. En base a la información recogida y a la experiencia acumulada por los profesores, se toman decisiones que se podrán aplicar en los cursos siguientes con el fin de mejorar la asignatura. Las mejoras más relevantes son las siguientes

- Coordinación con los profesores de las asignaturas previas para trasladar esta metodología de trabajo en el laboratorio y así que los alumnos adquieran mayor experiencia en ella, antes de cursar la última Experimentación.

- Recomendar el inicio del proyecto en la semana 3 del cuatrimestre y un grado de avance del proyecto del 60-70 \% al alcanzar las semanas 6-7 (tutoría grupal), para que no se les acumule el trabajo al final.

- Respecto a la disponibilidad de los profesores, reducir el tiempo de respuesta de la cita por parte del profesor, nombrando un único interlocutor de cada grupo con el profesor para concertar la misma. Advertir a los alumnos de los periodos de mayor concentración de tutorías y el incremento del tiempo de respuesta en esos periodos al solicitar tutorías.

Tabla 1.2. Aspectos positivos y negativos del ABP derivados de la reunión de "focus-group"

\begin{tabular}{|c|c|c|}
\hline Tema & Aspectos positivos & Aspectos de mejora \\
\hline Contenidos & $\begin{array}{l}\text { Alto nivel de satifacción con el } \\
\text { hilo conductor de las prácticas } \\
\text { Planteamiento diferente y } \\
\text { motivador frente a las } \\
\text { asignaturas previas de } \\
\text { experimentación }\end{array}$ & --- \\
\hline $\begin{array}{l}\text { Informes de } \\
\text { prácticas }\end{array}$ & $\begin{array}{l}\text { Prefieren la metodología } \\
\text { aunque les cuesta adaptarse a } \\
\text { realizar el informe en el } \\
\text { laboratorio }\end{array}$ & $\begin{array}{l}\text { Algunas prácticas requerían dedicación } \\
\text { adicional fuera del laboratorio lo que } \\
\text { incrementa su carga de trabajo }\end{array}$ \\
\hline \multirow[t]{2}{*}{ Tutorías } & $\begin{array}{l}\text { Necesarias para hacer el } \\
\text { proyecto }\end{array}$ & $\begin{array}{l}\text { Dificultades de coordinación con el } \\
\text { profesor para concertar cita: } \\
\text { disponibilidad y tiempo de respuesta }\end{array}$ \\
\hline & & $\begin{array}{l}\text { Dificultad de coordinación de los } \\
\text { alumnos }\end{array}$ \\
\hline \multirow[t]{2}{*}{ Proyecto } & Es necesario trabajar en equipo & Necesario más tiempo para su desarrollo \\
\hline & $\begin{array}{l}\text { Aprender a organizarse el } \\
\text { tiempo y la carga de trabajo }\end{array}$ & $\begin{array}{l}\text { Dificultades para organizarse el trabajo y } \\
\text { el tiempo, sobrecarga al final del } \\
\text { semestre }\end{array}$ \\
\hline
\end{tabular}


Lecciones aprendidas a partir de la experiencia de la aplicación del aprendizaje basado en proyectos en el Grado en Ingeniería Química de la UPV

Finalmente, cabe señalar que, aunque algunos alumnos sugirieron desglosar el proyecto con entregables a lo largo del semestre para evitar la acumulación del trabajo al final del mismo, otros pidieron no hacerlo para poder aprender a regular la carga de trabajo y a planificarse y organizarse de cara a proyectos futuros. Los profesores compartimos el segundo enfoque, dado que les hace ser más responsables de su propio aprendizaje y fomenta la planificación y organización del tiempo. Por ello, no se realizarán cambio a este respecto. No obstante se les advertirá en la presentación de la asignatura de las consecuencias de no realizar un trabajo progresivo: prisas, desencuentros, mayor presión, menor calidad del trabajo, dedicación exclusiva al proyecto en las fechas próximas a la entrega, toma de decisiones precipitada, ausencia de supervisión por parte del profesor, etc.

\section{Conclusiones}

-Para el éxito de la implementación del ABP en asignaturas experimentales, es importante relacionar todas las prácticas de laboratorio bajo un mismo hilo conductor y que éste tenga su traslado a un proyecto.

-La organización de la asignatura debe permitir una dedicación mayoritaria del tiempo fuera del laboratorio a la realización del proyecto, por lo que se recomienda la realización de las memorias de prácticas en paralelo a la sesión de laboratorio.

-Las tutorías son necesarias y útiles para el desarrollo del proyecto. Es recomendable una sesión obligatoria a modo de tutoría grupal como punto de control del desarrollo del trabajo. El profesorado debe tener flexibilidad y disponibilidad suficiente para concertar las citas con los alumnos y debe saber que implementar el ABP supone una sobrecarga de trabajo considerable en forma de sesiones de tutoría. Los alumnos deben ser responsables y organizados para distribuir la carga de trabajo a lo largo del semestre y no concentrar el desarrollo del proyecto en las semanas previas a la entrega.

\section{Referencias}

Arnal, J.M., Sancho, M., García-Fayos B. (2012). Evaluation by the students of the implementation of project oriented learning in an experimental subject of chemical engineering. En: International association of technology, education and development (editor), International Technology, Education and Development Conference (INTED 2012) (pp. 1-6). Valencia: IATED 
Blumenfeld, P.C., Soloway, E., Marx R.W., Krajcik, J.S., Guzdial, M., Palincsan, A. (1991). Motivating Project-based leaning: sustaining the doing, supporting the learning. Educational Psychologist, 26 (3\&4), 369-398.

De Miguel, M. (2005). Modalidades de enseñanza centradas en el desarrollo de competencias.Orientaciones para promover el cambio metodológico en el EEES. Recuperado de: https://www2.ulpgc.es/hege/almacen/download/42/42376/modalidades_ensenanza_competencias _mario_miguel2_documento.pdf

Kuntalp D.G., Demir G.K., Akay O. (2010) A Case Study of Modular-Based Project Oriented Learning in Electrical Engineering. En: Lytras M.D. et al. (editores) Technology Enhanced Learning. Quality of Teaching and Educational Reform. TECH-EDUCATION 2010. Communications in Computer and Information Science, vol 73. Springer, Berlin, Heidelberg

Peñas, F.J.; Barona, A.; Elías, A.; Olazar, M. (2006). Implementation of industrial health and safety in chemical engineering teaching laboratories. Journal of Chemical Health \& Safety, March/April, 19-23.

Sancho, M., Arnal, J.M., Santafé, A., Cuartas B., Martínez F., García-Fayos B. (2007). A new methodology in an experimental subject of chemical engineering. En: International Association of Technology, Education and Development (editor), International Technology, Education and Development Conference (INTED 2007) (pp. 300). Valencia: IATED

Sancho, M., Arnal, J.M., García-Fayos B. (2009). Application of project based learning in experimental subjects of chemical engineering. En: International Association of Technology, Education and Development (editor), International Technology, Education and Development Conference (INTED 2009) (pp. 2848-2854). Valencia: IATED

Sancho, M., García-Fayos B. Arnal, J.M. (2017). Methodological change in an experimental subject of chemical engineering degree: project learning based on laboratory practice. En: International Association of Technology, Education and Development (editor), International Technology, Education and Development Conference (INTED 2017) (pp. 6390-6396). Valencia: IATED 